\title{
Possibilities of Using Augmented Reality in the Health Field
}

\author{
Madalina Tofan \\ madalina.stefan@unitbv.ro \\ Gabriel Bratucu \\ gabriel.bratucu@unitbv.ro \\ Gheorghita Dinca \\ gheorghita.dinca@,unitbv.ro \\ Transylvania University of Brasov, Romania
}

\begin{abstract}
The augmented reality, as technology applied in medicine, meets the expectations of potential users, bringing them a lot of experience. The diversity of the use of augmented reality applications explains the need to develop and broadly apply the new concepts, and for this reason the authors conducted a theoretical research on the possibilities of using the augmented reality in the sanitary field. The advantages of using the new technologies are mentioned in the present paper, and consumer needs are described based on the theoretical research.
\end{abstract}

Keywords: augmented reality, healthcare system, cost reduction, education, healthcare development.

JEL Code: I1, O3, I2,

\section{Introduction}

The augmented reality, as a technology applied in medicine, meets the expectations of potential users, bringing extra experience to students at the faculties of medicine, but also helping physicians renowned in solving complicated cases. Augmented reality applied in healthcare can be an effective way to help patients in better understanding certain medical procedures and even more, help patients overcome certain medical problems through non-invasive methods.

The diversity of augmented reality applications explains the need for development and and the broad application of newly emerging concepts. Along with advances in optics, information systems and surgical instruments, augmented reality's demand for medicine is being studied vigorously.

Augmented reality has an important role in many medical applications, especially those using the laparoscope, endoscope, or catheterization interventions. Surgery changes from open procedures to minimally invasive approaches. Augmented reality technology has great potential to support this change and is becoming more and more important. The biggest advantage is to visualize the region/point of interest, such as tumors, blood vessels and nerves that are often invisible or hidden to direct vision ${ }^{97}$.

The impressive development of medical imagistics technology over the past decades has provided for physicians a growing amount of anatomical and functional data that is patientspecific. In addition, the increasing use of real-time non-ionizing images, especially ultrasound and optical imaging during surgical procedures, has created the need to design and develop new viewing and display technologies that enable physicians to make full use of rich sources preoperative heterogeneous and intraoperative data. In the 1990s, augmented medical reality was proposed as a paradigm that brings new perspectives of visualization and interaction ${ }^{98}$.

97 Ho-Gun Ha and Jaesung Hong, Augmented Reality in Medicine, Hanyang Med Rev, 2016 Nov, 36(4), pp.242-247

98 Sielhorst, T., Feuerstein, M., Navab, N., Advanced Medical Displays: A Literature Review of Augmented Reality, Journal of Display Technology, Volume: 4, Issue 4, Dec. 2008, pp. 451 - 467 


\section{The need for augmented reality in medicine}

One of the greatest benefits of augmented reality is the ability to demonstrate and clearly visualize complicated concepts. The competitive landscape between manufacturers and suppliers of medication/medical equipment means that small benefits can bring big gains. For sales reps competing for a physician's attention, the ability to quickly demonstrate the benefits of a new drug through augmented reality can be extremely beneficial. For example, viewing a 3D organ on a doctor's desk and demonstrating the effect of that medicine has a greater impact than a simple picture. Health professionals could use scanning to see the effects of a disease in 3D to find out how treatment works, to see potential cost savings and much more.

Healthcare centers, such as hospitals and treatment centers, have increased care due to the prevalence of superior technologies and doctors' expertise, but now there is an increase in circulation towards more flexible and mobile care. Why? Access to healthcare in rural areas may have poor medical outcomes. It is often difficult for patients or caregivers to come to a healthcare office, especially when dealing with restrictive work or child care obligations.

In response to these new needs, there has been an increasing interest and investment in telemedicine. But technology has not made telemedicine realistic, except for diagnosing and treating common affections. But this can change with the development of augmented reality and virtual reality. These technologies have the potential to eliminate the boundaries that divide offices, hospitals and homes, and to redefine how medical specialists provide healthcare to patients with chronic conditions.

From all of the above, we can draw some economic and human needs:

- The correctness of a medical diagnosis by non-invasive or minimally invasive methods.

- Convenience of diagnosis identification.

- Accessibility of health services in rural areas.

- Prevention.

- Health education.

- Reduced costs of the diagnosis and reduced treatment costs.

- Simplified patient-physician communication.

- Professional development.

- Performance.

- Evaluation.

Since the 1990s, augmented reality-based technologies have been on the rise, identifying more and more user needs, and trying to find the best ways to meet their needs. There is a multitude of applications that are already being used in the developed healthcare systems around the world, and they are both useful to doctors and patients.

Augmented and virtual reality are promising inventions with many potential applications in the hospital environment. Studies to date demonstrate utility, but there is a bigger need of well-controlled studies to demonstrate clinical and cost effectivenes ${ }^{99}$.

One challenge is to develop or adapt computer systems based on expert support in order to be used by consumers. Most of these systems were originally developed to provide advice for clinicians on diagnosis or disease management, but they also could cover certain needs of healthcare clients. Computer-based applications are developed to help doctors integrate patient preferences (values) with scientific evidence, patient history and local constraints. These systems also help patients to make treatment or screening choices based on their preferences for different outcomes ${ }^{100}$.

\footnotetext{
99 Dascal, J. et al, Virtual Reality and Medical Inpatients: A Systematic Review of Randomized, Controlled Trials, Innov Clin Neurosci, 2017,14(1-2), pp. 14-21.

100 Eysenbach, G., Consumer health informatics, BMJ Publishing Group, 2000, pp.1713-1716, available at: https://www.ncbi.nlm.nih.gov/pmc/articles/PMC1127483/\#
} 


\section{Discussions}

Through immersive environments, clinicians are more likely to expose patients to fearful stimuli over traditional techniques, providing greater efficacy in treatments and resulting in significant improvements in the overall welfare of patients. In addition, the mobilization of healthcare services for smartphones and other devices facilitates the migration of services beyond the walls of the traditional doctor's office, in the homes and everyday life of those who need it the most. This also implies an important intervention in the economic environment, which is implicitly influenced by the emergence and use of new technologies.

Surgical simulators have already proved to be extremely precious in training students and novice doctors, which demonstrates the interdisiplinarity of augmented reality in various areas in this case in the educational system with practical medicine application. By combining visual simulation with haptic technology, the student or young surgeon can virtually experience all the essential factors of a procedure that normally involves invasive techniques on a real patient or a corpse.

Also, a very important role of augmented and virtual reality technologies is as diagnostic mechanisms. By associating these methods with other ones such as MRI imaging, for example physicians can clearly establish the causes of a condition without the need of invasive or complicated procedures. These aspects reduce long-term costs and demonstrate their effectiveness in modern healthcare systems that focus on development and innovation.

Starting from digital systems that digital design on connected mannequins and simulated surgical devices to light carrier devices that provide medical knowledge based on vital signs monitoring, augmented and virtual reality vary depending on where and how they are used. In most cases, the only real limitation of augmented and virtual reality is human creativity. Any medical scenario could be simulated for learners/students/users. Cancer patients who are isolated during chemotherapy treatment can travel anywhere with a headset, provided that someone can make visuals rich enough to take the patient away. These technologies can make so much that it is essential that innovators be willing to address to healthcare systems and take benefit of this opportunity.

The first health field that has been influenced by the augmented reality has been medical education. Innovations in information technology, along with the low cost of hardware and software, will refine and enhance the efficiency, expertise and competence of the medical act in order to ensure patient comfort and an adequate and safe therapeutic approach ${ }^{101}$.

Along with virtual reality and interactive computer-generated environments, people can experience their problematic situations repeatedly, and learn through psychological evidencebased treatments how to overcome the difficulties ${ }^{102}$. Also, start-ups are currently using virtual and augmented reality technologies in order to train employees by helping them to familiarize with different backgrounds, in the end bringing benefits for both parties.

For both healthcare professionals and patients or other health care providers, an important role is to ensure the availability of skills and familiarity with modern tools, as well as a proper understanding of the context in which they interact. Cyberterapy has begun to make progress in treating a variety of disorders. However, there is still a need for the use of these techniques and the creation of accessible environments, the development of objective measurement tools, the need to adapt potential side effects, and the implementation of more controlled studies for the assessment of cyber resistance compared to therapies ${ }^{103}$.

101 Bălan, O., Moldoveanu,A., Moldoveanu,F., Morar,A., Virtual Reality in healthcare - Achievements, Problems and Trends, 2013 available at:

http://www.academia.edu/17251974/Realitatea_Virtuală_în_Medicină-Realizări_Probleme_şi_Tendințe

102 Freeman, D., et al, Virtual Reality in Assessing, Understanding and Treating Mental Health Disorders, 2017, available at:https://www.cambridge.org/core/journals/psychological-medicine/article/virtual-reality-in-theassessment-understanding-and-treatment-of-mental-health-disorders/A786FC699B11F6A4BB02B6F99DC20237 103 WiederholdIan, B., Wiederhold, M., Augmenting Behavioral Healthcare: Mobilizing Services with Virtual Reality and Augmented Reality, Digital Health, 2018, pp 123-137, disponibil la: 
One of the examples of augmented reality application in medicine refers to women's specific medical procedures, such as gynecological interventions, which often serve as sources of stress and anxiety for women, resulting in increased pain, decreased welfare, and decreased likelihood of perform the necessary examinations. In particular, new technologies have emerged as an effective tool in behavioural health, used to treat phobias, anxiety, and stress and pain management ${ }^{104}$.

In health field, augmented reality has been integrated in a series of applications. Surgery, anatomy, education and simulation are some of these. Augmented reality applications have been developed with varying success so far, but most agree that augmented reality will become better and will become a part of clinical care and education in next years.

At the International Healthcare Simulation Meeting (IMSH) in Los Angeles, California (the largest medical simulation conference) from January 2018, CAE Healthcare announced the CAE LucinaAR release together with Microsoft HoloLens as the first birth simulator from the world. It is the first augmented reality simulator for childbirth and includes both mother and fetal physiology. The learning module provides immediate assistance and feedback to physician movements and information about their effectiveness. It is obvious that the largest companies in the world devote an important part of their activity to research and development of augmented reality, which directly explains the economic implication.

Augmented reality applications, usable devices and mobile devices have shown tremendous potential in self-monitoring, diagnosis, and patient care. Augmented reality, in real light, offers digital images, sounds or other stimuli that give information about collected data, or add extensive detail to reality. From contact lenses that give visual indications to diabetics when glucose levels fluctuate dangerously to smartwatchs that use real-time communication to remind patients to take prescription drugs when they are nearby, there are already technologies in development that helps patients take control of their health through AR.

Augmented reality can also help healthcare providers to free themselves from fixed technologies that limit circulation and disrupt care. Based on stationary terminals for data entry, viewing medical records should not necessarily be a standard practice. For example, the goggles that use augmented reality with gesture-based interfaces, display information for patients and creates the hands-free opportunity which is extremely useful to suppliers. Given that technology, access to data and information processing are already at a advanced level, the next step is to bring meaningful information, even life-saving, in the attention of doctors. For example, if there is a complicated operation, there may be very little time to check if the patient has a specific type of allergy. So, instead of searching through documents or databases, the surgeon could see the relevant data on his AR screen in seconds. However, not only data, but other types of medical information, such as the location of veins or organs, could be projected into the environment, helping physicians in their activity.

Experts say that augmented reality will transform medical care by improving precision during operations, reducing medical errors, and also providing physicians and patients a better understanding of complex medical issues.

An example of economic need based on augmented reality is the UK National Health Service (the national healthcare provider in the UK) which trains thousands of nurses, doctors and employs about 1.5 million persons every year, which makes it the most important global employer. As a result, they are constantly looking for better ways to train their employees and achieve better learning outcomes. One problem they identified was that some areas were challenging for students if using conventional training methods that are both costly and timeconsuming. They needed a solution to accelerate the transfer of knowledge in key areas, reduce costs and improve the number of people who can be exposed to training, and augmented reality is the perfect answer to the problem. 
The combined market of augmented and virtual reality grows exponentially, as can be seen in the graph below:

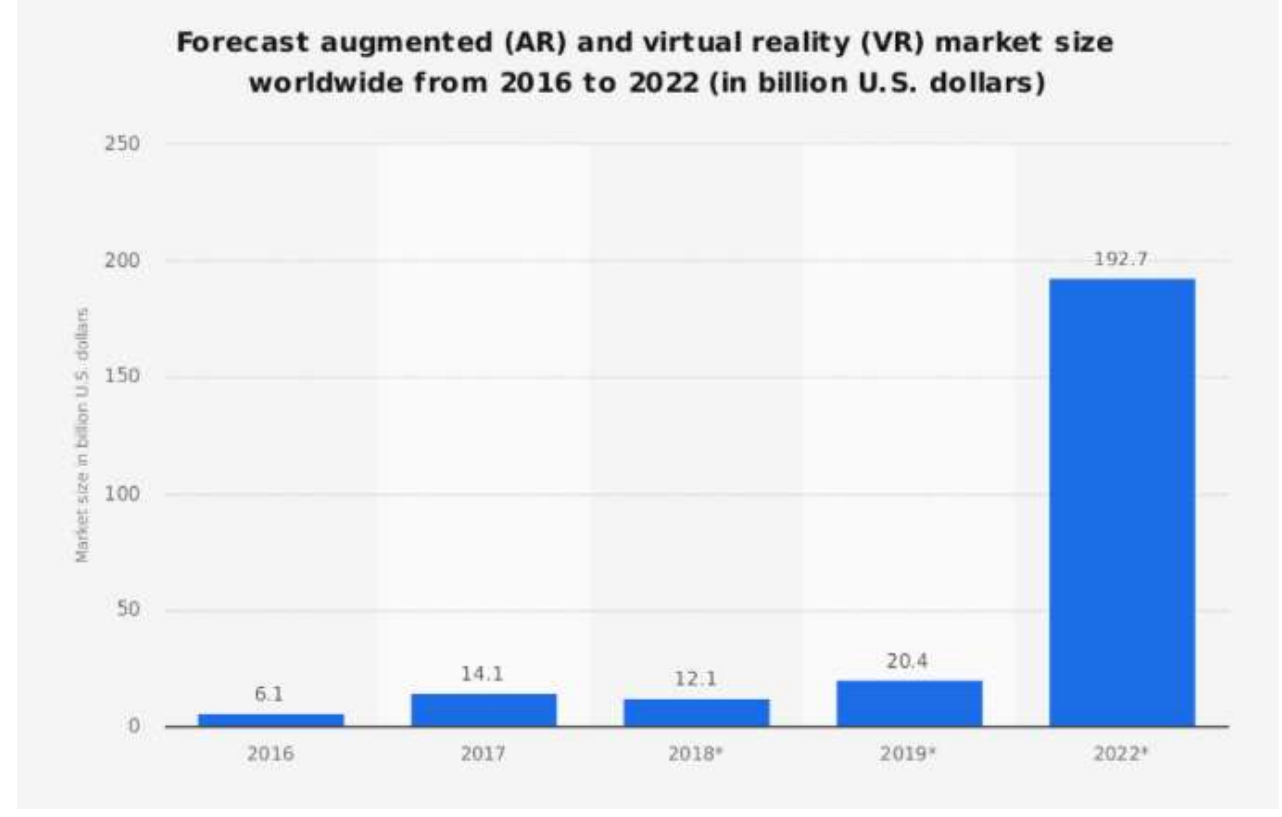

Figure 1. Market size forecast

Source: https://www.statista.com/topics/3286/augmented-reality-ar/

These data represent the image of the future, but for medicine and the healthcare systems these are a necessity, a natural approach to responding to the needs and expectations of the population.

Results published in 2018 by Perkins Coie International Law Firm as a result of the research conducted among new technology experts, reveal that health is ranked No. 3 in the interest of investment in the augmented reality interests (ranked first being gaming and ranked the second being education). The biggest impediment identified is user experience and the costs related to development of these tehnologies. The same study indicates that augmented reality will lower virtual reality in less than three years, and the biggest obstacles in developing new technologies from companies' point of view is data security and consumer privacy.

Certainly the gaming industry is vast and offering, but the satisfaction of using augmented reality in the healthcare field is far more important, relying on the image of a healthy population, but at the same time can lead to a reduction in the development time of new products, and also in testing them and implementing treatments.

Even if it is not easy, the expectations of healthcare customers have to intersect with the perceptions ${ }^{105}$, being a field in which the psychological implication is an important one. Although the healthcare systems provides concrete physical evidence, the real accumulated benefit by the consumer is an intangible item. ${ }^{106}$

\section{Conclusions}

Augmented reality technology needs to evolve, in order for all benefits to be tested and approved, and doctors need to buy the concept. The hardware must fit comfortably and securely with the practitioner's head. For some applications, the images must be as opaque as possible,

105 Mohan, K., Health Care Marketing, Discovery Publishing House, 2006, p.56

106 Kalenskaya, N., Gafurov, I., Novenkova, Marketing of educational services: Research on service providers satisfaction, Procedia Economics and Finance 5, 2013, International Conference on Applied Economics 2013, pp.368376 
while others will need to be more translucent. If the projected images are used for operative guidance, they must be positioned with extreme precision. There are a variety of technical challenges, but none of them is unthinkable. diverse:

The needs and expectations of potential users of augmented healthcare technology are

the prevention of certain diseases, which can easily be explained with the help of new technologies, bringing to users' attention the good practices in various fields;

learning as easy as possible by combining studied theoretical concepts and practice explained with the help of augmented reality, also benefiting from the opportunity of learning special and very serious cases;

ease of decision-making, ease of communication between doctors (physicians from different countries, facilitating remote exchanges of experience), ease of communication between doctors and patients (facilitating access to healthcare by the population regardless of the background);

ease of patient diagnosis by minimally invasive methods;

the behavioural change is induced by explicitly describing the reasons/benefits that accompany the cause;

easy pre and post-consultation patient and easy treatment assessment;

development of adjacent medicine and techniques through the variety of studies and experiments conducted, resulting in information which is available to all network users;

the interdisciplinary nature of the information that can be made available to users in order to help the professional development.

The advantages of using augmented reality in medicine are clear and incontestable. Worldwide, the emergence of the results of its use will not be delayed, and the desired behavioural changes will be achieved in a much shorter time, with minimal effort on the part of users, but with the maximum possible effect. The augmented reality is ready to anticipate population and sanitary systems problems and also their needs in order to contribute to the reduction of the existing gaps between the markets through the ease of use, respectively the reduction of the inequalities between the categories of consumers, patients and users.

\section{Acknowledgement}

This work was supported by a grant of the Romanian Ministry of Research and Innovation, CCCDI - UEFISCDI, project number PN-III-P1-1.2-PCCDI-2017-0800 / 86PCCDI/2018 FutureWeb, within PNCDI III.

\section{References}

1. Bălan, O., Moldoveanu,A., Moldoveanu,F., Morar,A., Virtual Reality in Healthcare - Achievements, Problems and Trends, available at: bttp:// wmw.academia.edu/17251974/Realitatea_Virtuală_in_MedicinăRealizări_Probleme_si_Tendinţe

2. Dascal, J., Reid, M., IsHak, W., Spiegel, B., Recacho, J., Bradley, R., Danovitch, I., Virtual Reality and Medical Inpatients: A Systematic Review of Randomized, Controlled Trials, Innov Clin Neurosci, 14(1-2), 2017, pp. 14-21.

3. Freeman, D., Reeve, S., Robinson, A., Eblers, A., Virtual Reality in Assessing, Understanding and Treating Mental Health Disorders, 2017, available at: https:// www.cambridge.org/core/journals/psychologicalmedicine/ article/virtual-reality-in-the-assessment-understanding-and-treatment-of-mental-health-

disorders/A786FC699B11F6A4BB02B6F99DC20237

4. Ho-Gun Ha and Jaesung Hong, Augmented Reality in Medicine, Hanyang Med Rev, 36(4), 2016, pp. 242-247, available at

5. https: / synapse.koreamed.org/search.php?where $=$ aview \&id $=10.7599 / \mathrm{hmr} .2016 .36 .4 .242 \&$ code $=0130$ HMREvmode $=$ FULL

6. Mihăescu-Pinția, C., Panait, C., Geană, A., Assessing Health Needs, the Starting Point for Policies and Effective Interventions, Management In Sănătate XXI/2/2017, pp. 4-8 
7. Sielhorst, T., Feuerstein, M., Navab, N., Advanced Medical Displays: A Literature Review of Augmented Reality, Journal of Display Technology, Volume: 4, Issue: 4, Dec. 2008, pp. 451 - 467

8. Várquez, J., Vaca, V., Wiederhold, B., Miller I., Wiederhold, M., Virtual Reality Pain Distraction During Gynecological Surgery-A Report of 44 Cases Pages 12-16, 2017, available at: http:// synergypublishers.com/downloads/sruv5a2/

9. Wiederhold, B., Riva, G., Fullwood, C., Smith, A., Kirwan, A., A Healthy Mind in a Healthy Virtual Body: The Future of Virtual Reality in Health Care, 2017, available at bttps:/ / docs.google.com / viewer? $a=v$ Epid $=$ sites $\Theta s r c i d=Z G V m Y X V$ sdGRvbWFpbnx1bmljYXROLWI0L WFyY3R0LXR/YW1 qLWV kaXRpb24tYmFja3V wfGd4OmM3ZTY4Nzg3ODRkNmJjMg

10. WiederholdIan, B., Wiederbold, M., Augmenting Behavioral Healthcare: Mobilizing Services with Virtual Reality and Augmented Reality, Digital Health, 2018, pp 123-137, disponibil la: bttps:/ / link.springer.com/ chapter/10.1007/978-3-319-61446-5_9

11. https:// wmw.cae.com/news-events/press-releases/cae-healthcare-releases-cae-lucinaar-first-childbirthsimulator-with-augmented-reality-for-clinical-team-training/

12. bttps:// wmw.extremetech.com/extreme/267078-vr-and-now-ar-medical-solutions-are-gaining-ground-inhospitals

13. bttps:/ / www.medicaldesignandoutsourcing.com/ how-ar-vr-devices-boosting-medicine/

14. bttps:// medicalfuturist.com/top-9-augmented-reality-companies-bealthcare

15. https://sciencebasedmedicine.org/augmented-reality-in-medicine/

16. https:// www.nbcnews.com/mach/ science/augmented-reality-lets-doctors-peer-inside-body-never-ncna847691

17. https://jumpsimulation.org/research-innovation/our-blog/2018/january/augmented-reality-in-health-care

18. bttps: / / www.eonreality.com/portfolio-items/angmented-reality-medical-diagnostics / ?portfolioCats $=609$

19. https:// www. bealthimaging.com/topics/imaging/rsna-2017-virtual-augmented-reality-may-revolutionizemedical-imaging

20. bttps:// www.forbes.com/sites/jenniferbicks/2017/09/20/using-virtual-and-augmented-reality-in-medicaldiagnosis-treatment-and-therapy/\#3be5dd63c4bc

21. bttps:/ / www.medlife.ro/ ochi-lenes-tratament-realitate-augmentata, https:// www.psychologicalscience.org/news/releases/angmented-reality-technology-could-belp-treat-lasy-eye.html.

22. https:// hbr.org/2018/03/ how-augmented-reality-will-make-surgery-safer

23. https:// www.blippar.com/blog/2018/05/18/3-benefits-of-augmented-reality-in-healthcare

24. https:// www.medtechimpact.com/ innovative-wellness-medicine-on-the-move-ar-vr-gaining-ground/

25. https://www.sciencedirect.com/science/article/abs/pii/S000145751730088X

26. https:/ / www.medicalplasticsnews.com/ news/technology/why-ar-and-vr-are-set-to-transform-the-medicaldevice-sector/

27. https://www.perkinscoie.com/en/news-insights/index.html?news_type_id=1000784\&start $=0$ \&ount $=5$ 